\title{
THE REVERSE MODELLING REALIZED BY DIGITAL SCANS AND FOTOMODELLAZIONE: BASED ON POST-PROCESSING COMPARED SEVERAL NOTEWORTHY EPISODES CONTAINED IN THE BASILICAL COMPLEX OF SAN LORENZO FUORI LE MURA IN ROME
}

\author{
M. La Mantia ${ }^{\text {a }}$ F. Lanfranchi ${ }^{\text {b }}$ \\ a,b Dipartimento di Storia, Disegno e Restauro dell'Architettura, “Sapienza” Università di Roma, Italy - \\ (mariella.lamantia,fabio.lanfranchi)@uniroma1.it
}

Commission V, WG V/4

KEY WORDS: Reverse Modelling, Fotomodellazione, Post-Processing, Basilica di San Lorenzo flm in Roma

\begin{abstract}
:
In the last decade, the subject area of the survey, and also that of representation, have undergone major changes with the introduction and use of advanced technologies that have revolutionized both the operational processes dedicated to the withdrawal of the measures that those graphic display data in terms of return and performance. The three-dimensional vision deriving from direct observation, or photogrammetric instrumental with the application of images, constitutes the most complete display means of the formal, morphological and material aspects of the detected object. 3D modeling offers many advantages, such as managing even the most complex geometric surfaces and its use is investing many sectors: in particular in the field of architecture serves many purposes including documentation, representation, diagnostics, materials and degradation, the enhancement and conservation of the property, etc.. The laser scanner technology together with that of fotomodellazione, is aimed to the acquisition, modeling and rendering threedimensional (3D) surface observed physical. The laser system to scan, as well as that of photographing, provides as a direct result of the measurement session a set of three-dimensional coordinates, in a reference system linked with the instrument. . The high amount of sampled data, in the order of thousands per second, allows to obtain a high level of detail offering an acquisition that could be called pseudo-continuous. In this context, there is a complex major project work on the Basilica di San Lorenzo flm in Roma, whose first partial results have recently been published - in the course of an activity which has been carried out to study and research for the establishment of procedures for processing the most appropriate and valid to be applied during post-processing for the eventual realization of a proper three-dimensional model. This paper will illustrate, through the analysis of some significant episodes of the Basilica, as the criteria adopted for the choices made at this stage have been crucial to the final outcome.
\end{abstract}

\section{INTRODUCTION}

The three-dimensional vision resulting from photogrammetric surveys and/or with the application of digital images, constitutes the most complete display means of the formal, morphological and material aspects of the detected object. The model provides three-dimensional shapes, colors, sizes element with complexity factor which is directly proportional to its application, to the time and cost of acquisition of the same. Their exhaustive graphic-communication makes them easy to understand and it shows their fundamental and irreplaceable value. Today, through new digital technologies, represented by the laser scanner and the fotomodellazione, you can, in an almost totally automizzata, create pseudo-three-dimensional models directly from measured data, with different processes of transformation and union representation, governed by algorithms diversified each program. The laser system to scan, as well as that of photographing, provides as a direct result of the measurement session a set of three-dimensional coordinates, in a reference system linked with the instrument, for a very high number of points that are hit by the laser beam or acquired through photography, the cloud of points generated in this way seemingly describes the outer surface of the scanned object. The high amount of sampled data, in the order of thousands per second, allows to obtain a high level of detail offering an acquisition that could be called pseudo-continuous. Within an articulated and complex major project work on the basilica of San Lorenzo outside the walls, - whose first partial results have recently been published - was carried out, in parallel, an activity of study and research for the definition of the procedures of processing more pertinent and to be applied during the phase of post-processing for the eventual realization of a three-dimensional model.

\section{CASE STUDIES *}

2.1 Brief historical evolution of the presbytery of St. Lawrence Outside the Walls Basilica in Rome.

The scope for today's presbytery area of the Basilica of St. Lawrence Outside the Walls in Rome is, in the current floor plan, the nave of the old basilica built in the sixth century under Pope Pelagius II.

It was as a result of the work of radical renovation and expansion made in the thirteenth century by Honorius III that the orientation of the basilica, originally set with east access, was inverted, giving rise to today's spatial and functional conformation on two levels.

The current area of the presbytery, the perimeter of which is bounded at the top by four double angle pillars of masonry, is upbeat on the long sides north and south and on the short side of the apse to the east, from the Corinthian order capitals placed at the top of the giant columns whose bases are located at the level of the crypt. 
The Pelagian scope is also characterized by the presence of important fixed liturgical furnishings among which: the bishop's chair and the related "plutei" placed on the short east side, and the sculptural ensemble represented by the canopy and its altar, placed almost at the western limit of the presbytery area in complete and privileged visibility from the nave of the Basilica, where the walking level is intermediate between the crypt and the presbytery.

From bibliographic sources we learn that "plutei" - of which we ignore the actual original function and location - could form the old iconostasis which crowned the ancient choir abolished with the restructuring of the XIII century by Honorius III. Dating back to a previous period is the bishop's chair that, based on formal and decorative local canons typical of the XIII century, is believed to be made from the combination of the chair - the oldest element - and the espalier characterized on its top by a trefoil arch (figure 1). The emphasis and the unity of the whole composition is accomplished through the important base covered in slabs of porphyry. The architectural-sculptural ensemble made up of canopy and altar is, considering the date carved into the lintels that support the roof system, prior to the Honorius restructuring.
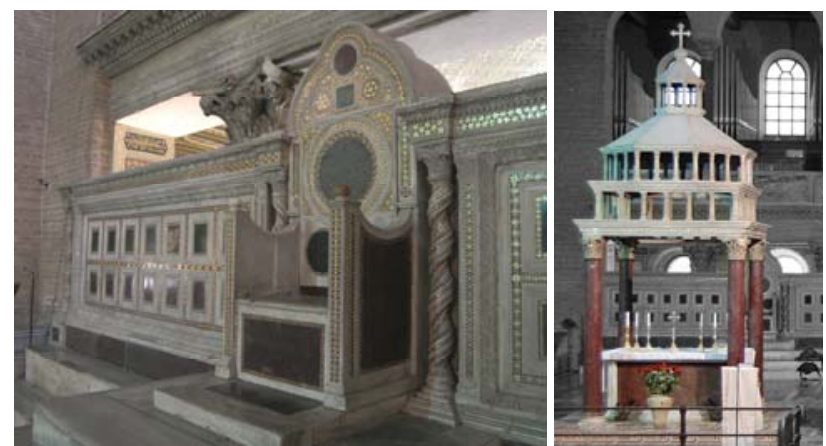

Figures 1,2. The bishop's chair and the ciborium.

The ciborium (figure 2), supported by 4 columns of red porphyry surmounted by lintels with support function for 2 additional orders of inlets distributed at the first level on a square base and at the second on an octagonal base, is completed with a pyramid based cover on top of which is placed a lantern topped by the cross.

The upper part of the canopy is probably owed to the works of restoration of the nineteenth century made by Vespignani who, in addition to restoring the second order of columns, replaced the previous dome cover. As for the chair and the "plutei" also the ensemble ciborium-altar is located on a base which in this case is coated with mosaics similar to those present on the entire pavement of the presbytery.

\subsection{The digital detection of bishop's chair and canopy}

The two episodes, already investigated by writers during the survey campaign aimed at the knowledge of the church and its monastery, have been an interesting field of application and comparison of digital post-processing procedures based on the development of point clouds acquired with range-based and image-based methods.

After an extensive investigation by bibliographic studies, followed a program of measurements, to be implemented both through direct and indirect detection techniques.

The choice of synergistically using consolidated operating modes such as the direct detection and the use of the total station on the one hand, and innovative techniques such as indirect acquisition by means of laser-scanner and digital photographic equipment, proved to be particularly effective as it has made possible a gradual, direct and yet comprehensive approach that is not limited to the partiality implied in sensory action limited to the sphere of visual perception.

\section{PRE-PROCESSING PHASE*}

\subsection{Acquisitions by active sensor (laser scanning) procedures and criteria}

As before mentioned, from the operational point of view, each indirect measurement was preceded by a campaign of acquisition of topographic points strategically targeted to the definition of a grid of trusted points which proved to be useful both during the registration of point clouds acquired by laserscanner, and in the operations of photographic straightening, in this case used for the subsequent control of the metric reliability of the models obtained in post-processing.

The particular configuration and at the same time the need of deepening in the definition of numerous and widespread elements - we refer for example to the particular textures of the mosaics relating to spiral columns placed at the ends of "plutei" as regards the furnishing of the bishop's chair, or architectural elements, even small and of particular value, such as the 4 marble capitals at the top of the columns of porphyry supporting the entablature of the canopy - naturally oriented choices for the positioning of the various measurement stations, both laser scanner and photographic, in turn recorded and referenced by the total station.

Leica devices have been used for the acquisitions, in particular a total station TCR 703 and a laser-scanner CYRAX HDS 3000. From Leica is also the software used for the management of the point clouds themselves.

Given the non-extended dimensions of the two distinct episodes, and their respective levels of detail and complexity, it has been planned a series of scans dedicated to the overall volumetric definition with acquisition values established in a grid of 3,5 x 3,5 mm "projected" to 4 meters.

In the case of the bishop's chair for example, characterized by a unique surface distinguished in two large rectangular marble elements (plutei) with dimensions of approximately 400 x 200 $\mathrm{cm}$, separate from the seat, also marble, ornamented as the sides from geometric motifs with polychrome mosaics, it was decided to make all the necessary scans for the proper framework and the minimization of the shadows, following a logic of equidistance both between the different scan bases and between bases and the investigated element.

The rhythmic setting of acquisitions has clearly made extremely smooth the step of joining the different point clouds, previously scheduled as feasible by direct recognition of homologous points rather than with the aid of a target. A process clearly chosen by virtue of common geometric patterns that characterize the entire architectural episode.

Finally, in relation to the acquisition phase, as the laser-scanner was integrated by a inside camera, for the acquisition of the correct RGB values it was preferred to apply to an external photographic apparatus - Canon digital camera defined in the next chapter - by which, once installed on a specific joint, it was possible to optimize - thanks to better optical and the controlled management of exposure times - the color rendering of captured images in low ambient lighting conditions.

As regards the management phase of the point clouds via software (Cyclone 7.4), once the different scans were joined, it was implemented a selective clipping operation with the aim of 
producing spatial areas as homogeneous as possible in terms of point density or loss of precision of the geographic position of the farthest points from the emission source. A substantial spatial definition in distinct areas of the individual clouds, an operation strategically targeted to prevent phenomena of redundancy of misleading points especially during the subsequent transformation into mesh (figures 3,4).

The resulting imprecision values calculated by the software during the unions, both partial and final, of both investigated architectural episodes were always kept well below the eligibility threshold taken of $0.009 \mathrm{~mm}$.

The encouraging numeric data returned by the algorithms of the point clouds management program have also been validated by the geometric and dimensional results given from the straightening operations that, as previously mentioned, were based on the acquisition by total station.

\subsection{Acquisitions by passive sensor (digital photographic images): procedures and criteria}

Once measurements implemented through active sensor instruments were completed, digital images aimed to the threedimensional reconstruction of the same episodes were acquired. Whereas one of the objectives of the experience was the comparison between the results arising from acquisition systems, active and passive, the choice of the capture method was oriented in favor of the photogrammetric fixed base acquisition technique; this technique can in fact offer spatial references with significantly lower inaccuracy margins than those obtainable by free stations.
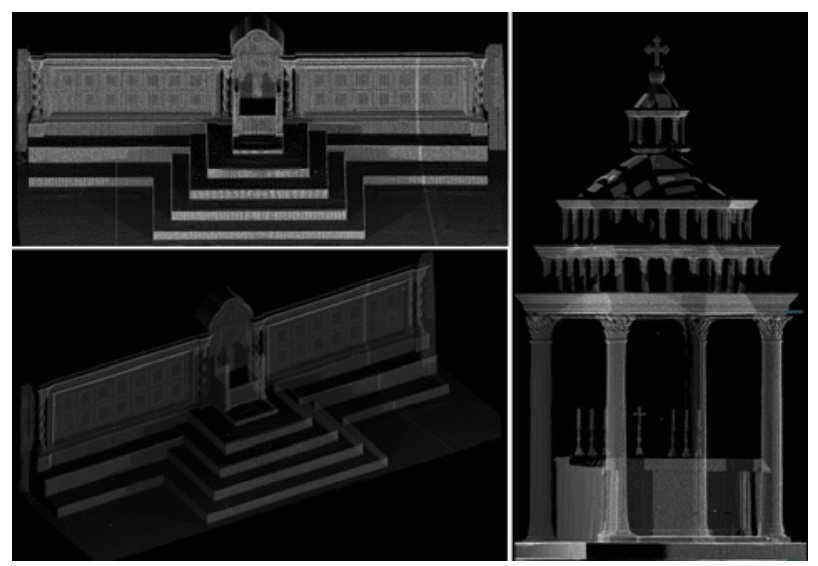

Figures 3,4. The images of the point clouds of the bishop's chair and the ciborium.

The opportunity to know the exact position of capture stations and of the investigated architectural elements, as well as the opportunity to establish exactly - by auxiliary diagrams - even before the acquisition phase overlap values of $60 \%$ minimum of the surface picture as required by the processing frames software, made it possible to implement a series of tests designed to control the data being processed.

Checks implemented by the same software Z-Scan (figure 5) which besides allowing the conform placement - thanks to the points of ground control station (Global Control Points) previously acquired with total station - of the model being processed in real spatial conditions of capturing, is also able to check the accuracy of the model being generated, interpolating the same points of georeferencing with those selected on photographic triplets and captured by total station.

With regard to equipment used in the acquisition and subsequent processing, it was used a Canon Eos 40D digital camera previously calibrated at the laboratories of Menci. Menci provided the Z-Scan system, consisting of a bar with constrained shooting distances installable on a tripod and proprietary software, as well as a Canon lens EFS 17-55 Ultrasonic.

The setting values of the device were adjusted to the values indicated by Menci both as regards the opening of the diaphragm set to F11, and for the focal length equal to $22 \mathrm{~mm}$, while as regards the sensitivity to exposure, in consideration to poor lighting, it was set to a value equal to 200 ASA.

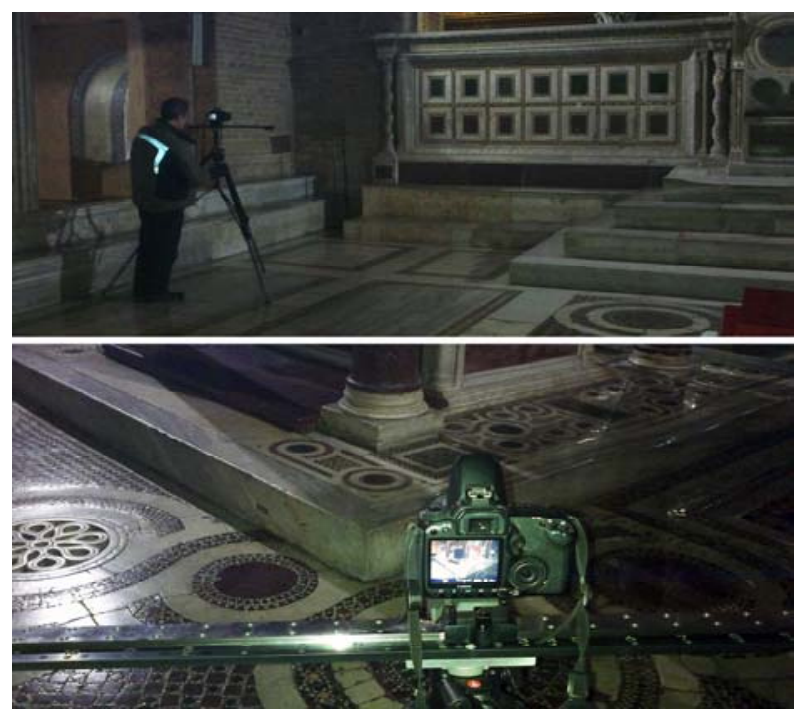

Figure 5. The images of acquisition technique with Menci

\section{POST-PROCESSING PHASE: CRITERIA AND OPERATING PROCEDURES**}

At this point, one thing is obvious and that is that the results of investigations, which must flow in two-dimensional or threedimensional graphic restitutions, which competes on the role of documenting and communicating the real substance configurative, structural and material - of the detected building, with the use of new methods of representation, don't emerge independently by digital processing of any computer, but must be extrapolated by operators. The acquisition techniques using laser scanner, for example, are not able to offer a product well definable as "representation", although they can well imitate it. The point cloud itself is only one set of numerical data from which to take measures for the dimensioning of the scanned items. The photo modeling also provides a pseudo-3d restitution which must necessarily be meshed and shaped so that the model is not designed to document reality with only informative purposes.

If in general is already valid the principle that sees as inappropriate the use of any tool or technique in an improper manner, is a fortiori counterproductive not to have the complete intellectual control of operations during both acquisition and management / manipulation of the digital data by the operator. In the scientific field of survey, the integration of different methods and techniques of post-processing results in the integration of procedures for the use of these techniques, which must meet three requirements: the reliability of the results, the repeatability of operations, the quality of restitution. As we said earlier, an objective of the paper is to present the most appropriate procedures to be adopted for post processing of point clouds acquired by image-based and range based instruments, derived from the comparison of the results, of the peculiarities of the used methods and of the issues detected in 
the two approaches, in order to test the greater or lesser validity of one with respect to another by comparing the results also in relation to architectural episodes with different morphologic and spatial connotations.

\subsection{Range based post-processing}

Referring to the previously described acquisition procedures, the data collected, after a first stage of pre-processing consisting in recording, cleaning and decimation of point clouds operation, may be further processed within the Cyclone software for the definition of mesh or exported for different types of file format (dxf, ptx, pts, ptg, ptz, coe, e57, xyz, tiff, etc.). The Leica Cyclone software allows the generation of triangulated surfaces (mesh) automatically, but it should be noted that the best results produced by the automatic procedure concern standardized elements of industrial production (structural steel and pipes), while the results produced in the case of architecture elements are very far from reality. The second option, the most traveled, is to export the data in the formats with which you can interface with various meshing and modeling software (Rapidform, Rhinoceros, 3d Studio, Geomagic, Reconstructor). You can also export data in ortho image (tiff) which generates, having previously set, according to specific cutting planes, the view, the range of colors, the size and density of point display, a raster element that allows only the tract vector restitution.

This technique is useful for two-dimensional development (plans, sections, elevations) but totally meaningless for the treatment and processing of surfaces. On the other hand, exporting the coordinates of object points coded with logical criteria, divided into punctual, linear and areal entities, displayable in various formats of which the simplest is a text file that contains in each row the three coordinates XYZ, allows post-processing and transformation. Data have been exported in dxf, ptx and xyz formats. The recorded export times were different for each type of format. At constant scanworld size, processing in ptx required a longer time with a weight of the resulting files that is twice the one obtained in xyz, with a substantial loss of information in the second case. The two exports from Cyclone are completely automatic and require, for a quantity of points of the order of 14 million, a period of time of about ten minutes. The size of the produced file is of the order of hundreds of Megabytes.

The dxf processing, on the contrary, except in cases where we considered the data on detailed elements of episodes (the capital and the base of the single canopy column, or the tortile column of the Chair) extrapolated from the episode itself, generated considerable problems for the amount of computing resources committed, sometimes so high as not to allow to complete the operation. The overall scanworld of the canopy consists of 342.288 points, while that of the Chair is made of 594.611 points. In the following table are resumed post processing data for different formats.

\begin{tabular}{lcccc|}
\hline \multicolumn{1}{c}{ Oggetto } & Formato & Numero Punti & Peso File (MB) & Tempo (minuti) \\
\hline Baldacchino &. $\mathrm{ptx}$ & 342288 & 35,2 & $10-15$ \\
Baldacchino &.$x y z$ & 34288 & 18,7 & $10-15$ \\
Cattedra &. $\mathrm{ptx}$ & 594611 & 59,3 & $10-15$ \\
Cattedra &.$x y z$ & 594611 & 17,2 & $10-15$ \\
Dettaglio Seduta &. $\mathrm{ptx}$ & 62303 & 6,89 & meno di 5 \\
Dettaglio Seduta &.$x y z$ & 62303 & 4,91 & meno di 5 \\
Dettaglio Colonna &. $\mathrm{ptx}$ & 26697 & 5,25 & meno di 5 \\
\hline Dettaglio Colonna &.$x y z$ & 26697 & 2,34 & menodi 5 \\
\hline
\end{tabular}

At this point, we have made a choice, which is conditioned by the way of managing files for meshing, opting for the xyz file for Rhinoceros and for the ptx file for Reconstructor. A small drawback happened reading the ptx format generated by a scanworld not structured, subjected to decimation. The illegibility of this by Reconstructor software forced to retrace the steps of pre-processing avoiding decimation (figure 6).

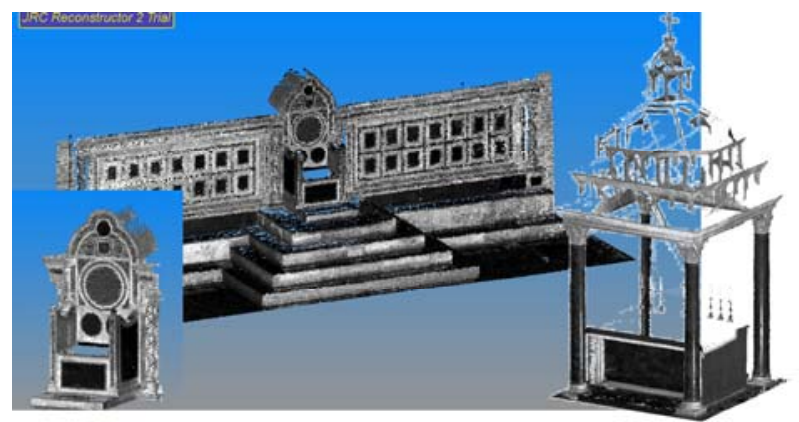

Figure 6. The visualitazion of the model elaborated with Reconstructor software

Importing the file xyz in Rhinoceros provides a first step in transforming the data, read by the software as a single object, in points, and then their meshing by suitably setting the values of "Densità Di Campionamento Più Rumore”, “Auto Aggiusta Griglia" and "Num Celle Contorno Griglia". In this way 450.000 mesh for the canopy and 900.000 for the Chair have been generated in the phase of triangulation. Even here it was not possible to operate in a single step the meshing of points in respect of large files, because of the excessive absorption of RAM but it was necessary to perform multiple steps at successive times. This has resulted, in some areas, in the generation of a two-fold stratification of the surface with significant inconvenience during texturing. Figure 7 shows the 3D model carried out automatically by the software. The simple visualization of the model allows to grasp the effect of smoothing of the surface with regard to the more complex situations (columns, capitals), besides the presence of empty zones in areas not sufficiently documented. The operation was repeated for each item. The import of the ptx file with Reconstructor software was easier to manage and it was possible to operate simultaneously throughout the whole architectural object. Skipping the settings related to the phase of meshing in this software environment, it has come to more than acceptable results.

\subsection{Image based post-processing}

The episodes in question, except for certain elements (capitals, spiral columns) present traditional canonical geometries consisting of flat and cylindrical surfaces. This facilitates, even in terms of time, the process of generation of the surface through the triangulation of the points. At the same surface area, the greater the number of points of the cloud, the greater the accuracy of this surface to the real one. The density of dots will be greater in areas with altitude varied and complex and curved areas, and less dense in flat areas. Therefore key elements in the three-dimensional reconstruction will be the choice of the correct spacing and best suited structure (TIN or GRID). From the data, that is the points, triangle vertices, edges, surfaces (triangular or square) are generated, as well as other extra points, called Steiner points, which help to create a better mesh. The next step is texturing, in which color images are mapped over the $3 \mathrm{D}$ geometric surface; then the RGB color values within the projected triangle are attached to its face. The problems associated with this process may result from possible radiometric distortions in the images, due to acquisitions with different cameras, from different positions and with different lighting conditions. 

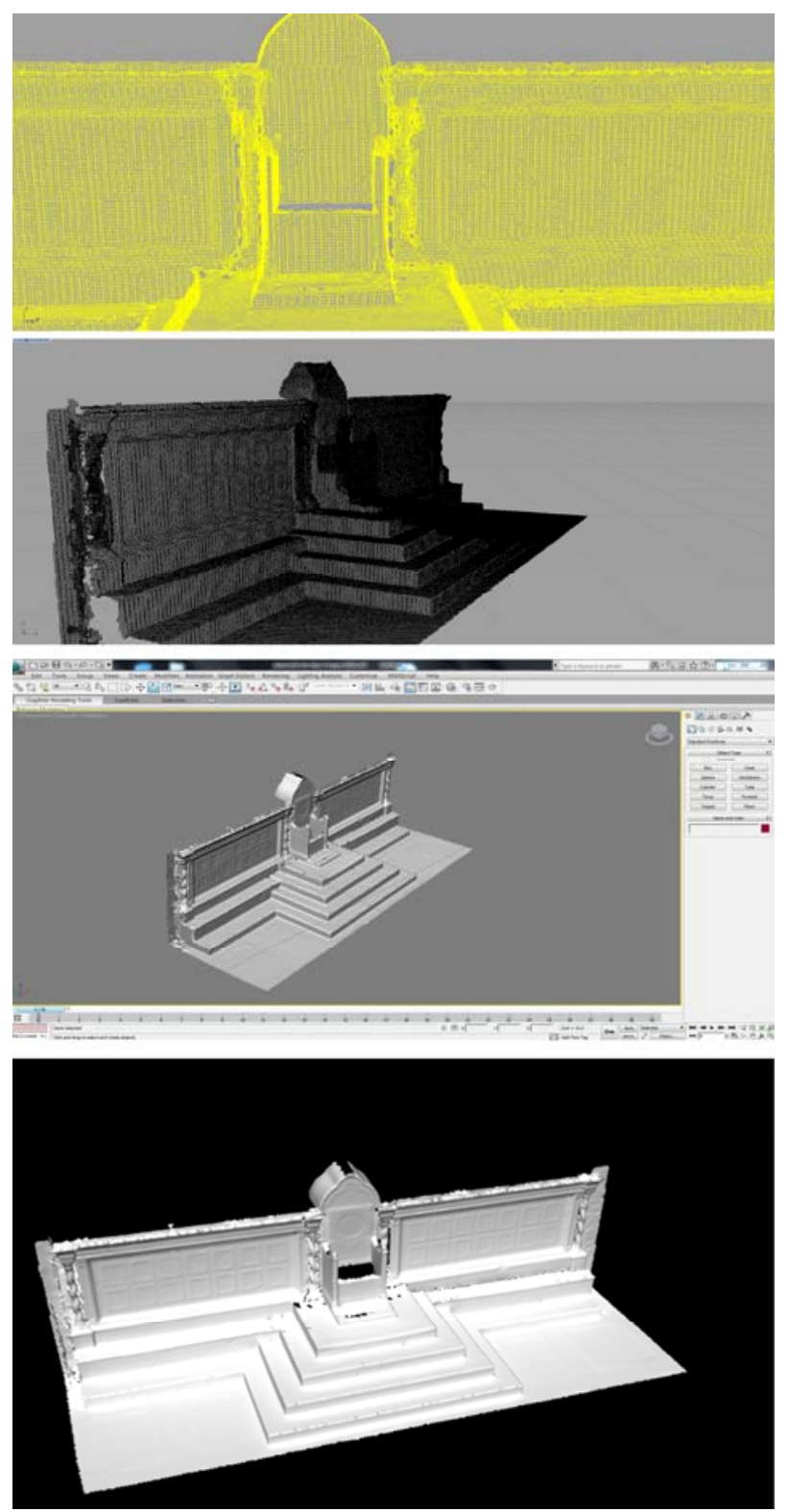

Figure 7. The images of the 3D model carried out automatically by the software. It's visible the effect of smoothing

The experiments carried out, in the case of clouds acquired with image-based method took into consideration systems both calibrated (Menci) and not calibrated (Autodesk 123D Catch). The difference in results obtained with Menci software and 123D Catch is due to several factors: firstly the different image matching algorithm used for the automatic generation of digital models of the surfaces after the phases of orientation, as well as the ability to process "post - editing" by the operator in order to eliminate errors due to incorrect matching, feasible in the first case but not in the second.

One of the fundamental processes of photogrammetry is the identification and measurement of conjugate points in two or more overlapping frames. In analog and analytical photogrammetry, the identification of conjugate points is carried out by an operator; digital photogrammetry tries to solve this problem automatically, through the process known as image-matching.

The process of image-matching, also known by the term automatic stereo matching or simply correlation, may use three methods: area-based matching in which the matching entity is formed by levels of gray (gray values) present in small areas of the two images; the feature - based matching, in which you select the characteristic elements (points, lines, edges) inone image and look for correspondents in the other; the relational matching, in which the process of image -matching is based on finding correspondences in existing relations (spatial, topological, radiometric, etc.) between collections of simple elements linked together. The phases of the process of image matching can be summarized in the identification of the entity matching in one of the images, in the recognition of the entity in the other image to which follows the processing of the 3D position of the entity in object space via the collinearity equations. The success of a good matching process depends on many factors.

The system of photogrammetric survey of Menci, even if more complex and laborious in the operations of recovery, because of its professional equipment of recovery, provides a greater level of precision and reliability. The realization of the point cloud is produced by three photographs, made with a special bar of recovery and a camera with calibrated optical. The software used is the Z-Scan . Since it is photographs, the points and their surfaces do not require the application of a texture as they already contain the necessary color information, reproducing, in this way, a 3D chart absolutely corresponding to reality.

The Bishop's Chair, unlike the Canopy, lends itself particularly well to the use of this technology. In fact, its almost linear trend allowed rapid use of instrumentation that did not need to be oriented differently from the position of parallelism with the object. Pairs of triplets for the resumption of the front and the side of the seat were performed The calculation results are shown in figures 8,9.

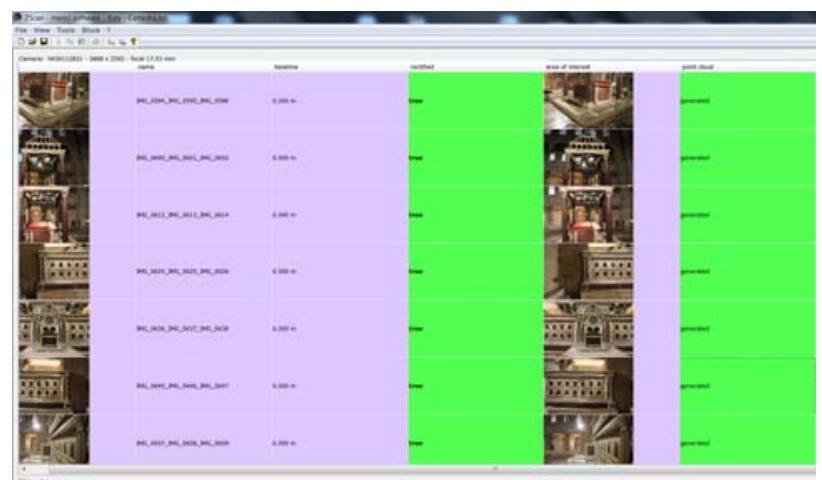

Figure 8. The window of the z-scan.

The generation of the surface from the point cloud for the Autodesk software 123D Catch is fully automatic, as there is no possibility to intervene. Autodesk 123D Catch, known in the Autodesk Labs as Project Photofly is a free application for reverse modeling with which you can create three-dimensional models of any object from a photo. The transformation is also feasible with non-professional photographic devices such as cell phones. The number of necessary shots is a function of the object to be detected. The ease of execution has oriented its use in the detection of some details of the canopy (capital and base). The absence of a priori given metric, since you are not using metric cameras, imposes, for the following scientific use of the produced elaboration, the capture of at least one measurement of the object or of the positioning, inside the area of photo recovery of a metric reference. The captured images are sent to an online server and are processed directly in automatic mode according to a certain algorithm. The process of calculating, depending on the complexity of the object, and then on the 
number of shots performed can take several minutes. The software also features the ability to process and save DWG format and obj. The 123D Catch method simultaneously considers all homologous windows in which there is a given point and compensates jointly the equation of the matching and the collinearity equations used as a constraint: therefore the image coordinates are determined by matching so that its rays projecting should meet in one and only one of the same object point. As you can see, the morphology of the object was not represented fully and accurately; the surface is smooth, without a precise definition of the forms, you feel the alternation of full and empty spaces that characterizes the capital, and is not possible to identify the individual elements that make it up, so the generation process cannot considered successful. Here, too, there is the negative effect of smoothing mainly due to the principle of area-based matching, which assumes that the homologous elements extracted are plans and, as mentioned above, to the lack of multi - frame matching. The final export can be made in different formats that record the description of the surface of a $3 \mathrm{D}$ object composed of triangles or a high number of polygons: obj and dwg, where the obj is associated with the file jpg (figure 10).
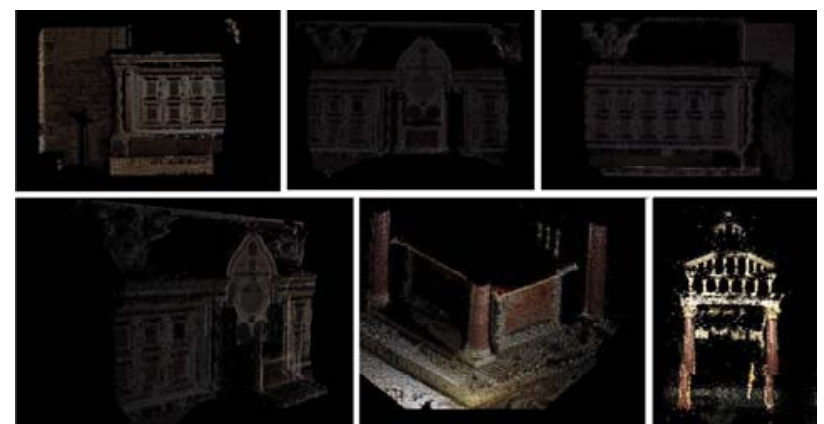

Figure 9. The image show the results with z-scan.

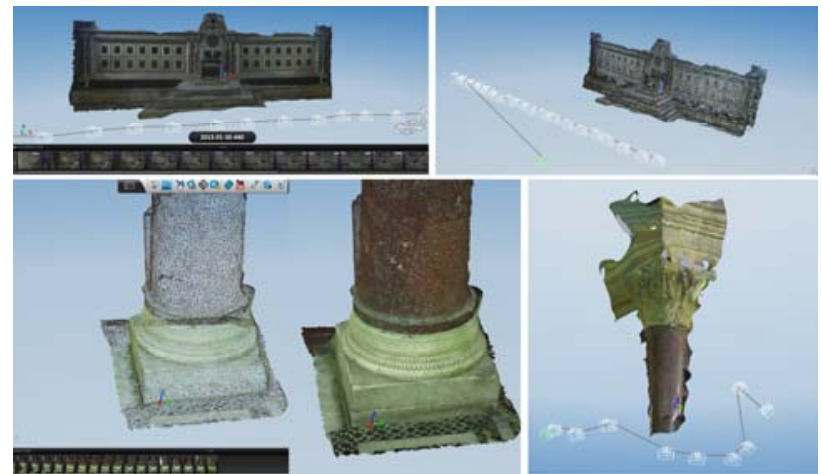

Figure 10. The image show the results with 123D cacth.

\section{CONCLUSIONS}

There are many issues related to the acquisition of a digital model from the data acquired with the range and image based methods, especially if that model is aimed not as much to documentation as to the scale model of the object, or in other words if the goal in our survey is the reverse modeling. The difficulties encountered are related to some extent to the limited field of action of the operator due to the automation of certain processes of processing, to the logistical conditions in which it is operated, and mainly to the inadequacy of available software to develop elements of a certain complexity. The 3D results achieved are the product of an integrated action between the two techniques considered under procedures that meet the criteria of efficiency and feasibility, even in terms of time and available instrumentation. This suggests a deepening of the scope of study widening the field to other software dedicated to the processing of this type of data.

In the case of photo modeling, for example, from the experiments carried out and the results obtained, we can say that while it is now possible to use digital cameras at low cost for the production of 3D models from images captured by non expert operators, on the other hand to achieve geometrically accurate and therefore reliable results, procedures and specific software tools are essential. The algorithms area-based leastsquares matching implemented in many cases are not sufficient to the generation of point clouds which then allow the creation of correct and accurate three-dimensional models. The methodology that makes use of the software 123 Catch has responded positively to the purpose only in a few cases and in any case not in those complexes.

From this point of view the laser scanning proves to be the most suitable product for the reconstruction of the complete geometry of the object, while the photogrammetry can help the understanding of the laser acquired data intervening in the phase of texturing the model with a photo-oriented object combining as well metric and descriptive data.

On the other hand, the point clouds produced by range-based methodology, require operator intervention in the management of the acquired data, as well as software capable of handling the modeling of irregular surfaces, so long lead times for processing and managing of considerable size files. This would suggest, in the case of simple planar surfaces, such as that of the bishop's chair, the use of photogrammetry for the general reconstruction of the episode and the laser scanner to get the more complex architectural details.

This contribution comes from the organic collaboration of both authors at each end have agreed to assign, respectively, the points marked by asterisks, (** Mariella La Mantia, *Fabio Lanfranchi).

\section{Bibliography}

Colombo, L., 1994: Dalla rappresentazione fotogrammetrica ai modelli raster tridimensionali. Bollettino SIFET, St.Vincent, Aosta.

Remondino, F. and Fraser, C., 2006: Digital camera calibration methods: consideration and comparisons. International Archives of Photogrammetry, Remote Sensing and Spatial Information Sciences, Vol.36(B5), Dresden, Germany.

AA.vV., Metodi e tecniche integrate di rilevamento per la realizzazione di modelli virtuali dell'architettura della città,

Ricerca COFIN 2004 - Coordinatore nazionale Mario Docci, Gangemi Editore, Roma 2007.

Cundari, C., Il rilievo architettonico. Ragioni. Fondamenti. Applicazioni. Aracne-Kappa Edizioni, Roma 2012.

Docci, M., Gaiani M., Migliari R. Una nuova cultura per il rilevamento, 2001. In "Disegnare. Idee, Immagini”, a. XII, n.23;

Cundari, C., Bagordo G.M., La Mantia M., Lanfranchi F., 2012. S. Lorenzo fuori le Mura a Roma. vol. 4, p. 1-182, Roma: Aracne Editrice S.r.l.

La Mantia M., 2011. Procedures and methods of digital modeling in representation didactics. In: AA.VV.. International Archives of Photogrammetry, Remote Sensing and Spatial Information Sciences. THE INTERNATIONAL ARCHIVES OF THE PHOTOGRAMMETRY, REMOTE SENSING AND SPATIAL INFORMATION SCIENCES, vol. XXXVIII-5/W16, Trento, 2-3-4 marzo 2011 